Bouncing droplets: a classroom experiment to visualize wave-particle duality on the macroscopic level

This content has been downloaded from IOPscience. Please scroll down to see the full text.

2016 Eur. J. Phys. 37055706

(http://iopscience.iop.org/0143-0807/37/5/055706)

View the table of contents for this issue, or go to the journal homepage for more

Download details:

IP Address: 131.211.105.88

This content was downloaded on 17/02/2017 at 15:16

Please note that terms and conditions apply.

You may also be interested in:

Probabilities and trajectories in a classical wave-particle duality

$\mathrm{Y}$ Couder and $\mathrm{E}$ Fort

The self-organization of capillary wave sources

Suzie Protière, Yves Couder, Emmanuel Fort et al.

Scattering theory of walking droplets in the presence of obstacles

Rémy Dubertrand, Maxime Hubert, Peter Schlagheck et al.

Wave-particle duality in classical mechanics

Alexander Y Davydov

Resonant and rolling droplet

$\mathrm{S}$ Dorbolo, D Terwagne, $\mathrm{N}$ Vandewalle et al.

Trajectory eigenmodes of an orbiting wave source

E. Fort and Y. Couder

Quantum properties of a which-way detector

J S Oliveira Filho, R Rossi Jr and M C Nemes

Build-up of macroscopic eigenstates in a memory-based constrained system

M Labousse, S Perrard, Y Couder et al. 


\title{
Bouncing droplets: a classroom experiment to visualize wave-particle duality on the macroscopic level
}

\author{
Pascal Sleutel ${ }^{1,5}$, Erik Dietrich ${ }^{1,2,5}$, Jan T Van der Veen ${ }^{3}$ and \\ Wouter $\mathbf{R}$ van Joolingen ${ }^{4}$ \\ ${ }^{1}$ Physics of Fluids, MESA+ Institute for Nanotechnology, University of Twente, The \\ Netherlands \\ ${ }^{2}$ Physics of Interfaces and Nanomaterials, MESA+ Institute for Nanotechnology, \\ University of Twente, The Netherlands \\ ${ }^{3}$ ELAN Institute for Teacher Training, University of Twente, PO Box 217, 7500 AE \\ Enschede, The Netherlands \\ ${ }^{4}$ Freudenthal Institute, Utrecht University, The Netherlands \\ E-mail: j.t.vanderveen@utwente.nl
}

Received 17 February 2016, revised 25 May 2016

Accepted for publication 30 June 2016

Published 27 July 2016

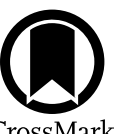

\begin{abstract}
This study brings a recently discovered macroscopic phenomenon with waveparticle characteristics into the classroom. The system consists of a liquid droplet levitating over a vertically shaken liquid pool. The droplets allow visualization of a wave-particle system in a directly observable way. We show how to interpret this macroscopic phenomenon and how to set up and carry out this experiment. A class of students performed single slit diffraction experiments with droplets. By scoring individual droplet trajectories students find a diffraction pattern. This pilot application in the classroom shows that students can study and discuss the wave-particle nature of the bouncing droplet experiment. The experiment therefore provides a useful opportunity to show wave-particle behavior on the macroscopic level.
\end{abstract}

S] Online supplementary data available from stacks.iop.org/ejp/37/055706/ mmedia

Keywords: walkers, wave-particle duality, classroom experiment, diffraction, droplets

(Some figures may appear in colour only in the online journal)

5 These authors contributed equally to this work. 


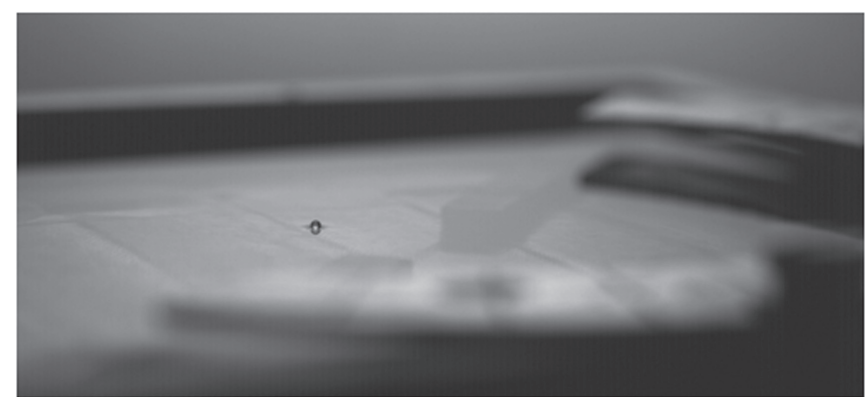

Figure 1. An image taken during the single-slit experiments, showing a walker, from supplementary movie 1 . Under the liquid surface, the structure forming the slit is visible. More detail about this setup can be found in figure 3 and the surrounding text.

\section{Introduction}

Over recent decades, quantum mechanics has found its way from laboratories and universities into high school classrooms. Even though it has not lost its gleam of mystery and difficulty, it has become part of the standard curriculum in high school physics education (Pol et al 2015). This poses the problem for teachers of explaining to their students phenomena that are not even fully understood by scientists. While the demonstration of appropriate experiments is a useful way to introduce and explain physics to students, few such experiments are available to help with understanding quantum mechanics. Many of the experiments conducted in the history of quantum mechanics are only feasible in a dedicated laboratory, so teachers commonly resort to the use of applets. Recently, Couder and coworkers demonstrated that millimeter-size oil droplets bouncing on a vibrating fluid bath can exhibit quantum-like behavior at a scale visible to the human eye. The experiment, working principle, and all of the physics behind it have been explained in detail (Protière et al 2005, Couder and Fort 2012, Perrard et al 2014). We establish that this experiment can be used in a classroom setting to visualize a wave-particle system, and certain other aspects of quantum mechanics. We begin by introducing the experiment and explaining the connection between the experiment and waveparticle duality, indicating both similarities but also limitations. We then describe the experimental setup, followed by a description of a pilot held with high school students. We conclude by presenting the results of this pilot, highlighting that this experiment can be executed in a classroom setting, by students.

\section{Physics}

\subsection{Walkers}

When a drop of liquid is dropped on a bath of the same liquid, one naturally expects the droplet to merge with the bulk of the liquid. However, when the bath of liquid is shaken in the direction perpendicular to the liquid surface, something surprising can happen: the droplet survives, bouncing over the surface of the liquid bath for a prolonged time. The vertical motion of the bath creates and maintains a thin layer of air between the droplet and the surface of the bath, preventing their coalescence. Something even more extraordinary happens when the droplet size, liquid type and shaking parameters are chosen just right: the bouncing droplet then creates waves at the surface of the bath, interacts with these waves, and is propelled 


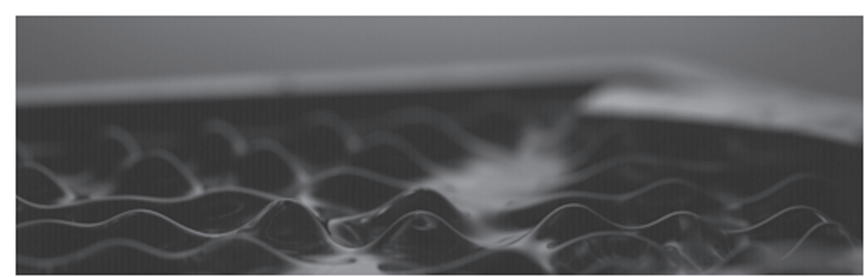

Figure 2. An image showing Faraday waves.

horizontally across the surface (Couder and Fort 2006). Such a droplet/wave combination is called a 'walker', a term that will be used throughout this work. An example of a walker is shown in figure 1. For a walker to exist, a coupling must be established between the shaking frequency of the bath and the droplet'(s) bounce time such that the droplet always hits the sloping side of the wave. This causes the droplet to be propelled over the surface with speed of $\approx 1 \mathrm{~cm} \mathrm{~s}^{-1}$ (Bush 2015).

Walkers can only occur when the liquid bath is vibrated at an acceleration close to the Faraday transition: when at rest, the liquid bath will have a flat, hydrostatic surface. Any disturbance of the flat interface will be counteracted by gravity and surface tension. However, if we shake the liquid bath following

$$
\gamma=\gamma_{m} \cos \left(2 \pi f_{0} t\right)
$$

in the direction perpendicular to the fluid surface, the liquid is accelerated up and down. In equation (1) $f_{0}$ is the oscillation frequency in $\mathrm{Hz}, t$ is the time in seconds, and $\gamma_{m}$ is the acceleration of the bath. For certain accelerations of the liquid bath (note that the acceleration is directly related to the frequency and amplitude of the harmonic motion), the liquid surface destabilizes and enters a state of parametric resonance. The standing waves that develop on the liquid surface are called Faraday waves, see figure 2 for a typical example of these waves. An everyday example of Faraday waves can be observed in a glass of wine, which is made to 'sing' by rubbing a wet finger over its rim. The acceleration of the liquid bath above which the Faraday waves appear is called the Faraday transition acceleration. At accelerations above this threshold, Faraday waves form on the entire surface. As it is the acceleration that determines if Faraday waves develop, we will describe the oscillatory motion of the liquid bath in terms of the acceleration, rather than by its frequency and amplitude.

The surface of the bath will be flat as long as it is oscillating at accelerations just below the transition acceleration. However, the impact of a bouncing droplet can be sufficient to trigger the formation of waves in the direct vicinity of the droplet. The developing waves are characterized by a wavelength, which can be calculated from the dispersion relation (Eddi et al 2011)

$$
\left(2 \pi f_{0}\right)^{2}=\left\{g k+\left(\frac{\sigma}{\rho}\right) k^{3}\right\} \tanh \left(k h_{0}\right),
$$

where $k$ is the wavenumber from which the wavelength $\lambda=\frac{2 \pi}{k}$ can be calculated, $g$ is gravitational acceleration, $\sigma$ and $\rho$ are the surface tension and density of the liquid, respectively, and $h_{0}$ is the depth of the liquid bath. The current experiment uses soybean oil, which has a surface tension of $\sigma=27 \mathrm{mN} \mathrm{m}^{-1}$, and a density of $\rho=0.92 \mathrm{~g} \mathrm{ml}^{-1}$ (Hammond et al 2005). Keeping in mind that the frequency of Faraday waves $f_{\mathrm{F}}$ is half that of the driving oscillation $f$ (Bush 2015), $f=50 \mathrm{~Hz}$ converts to a wavelength of $\lambda=4.45 \mathrm{~mm}$, which will be used later in the design of the experimental setup. 
With an acceleration below the transition value and without agitation of the surface, the surface will remain flat. However, as the droplet is bouncing repeatedly on the interface, it will trigger the formation of Faraday waves at regular intervals (at the moment of impact), creating a pattern of waves. Eddi et al (2011) simulated the wave patterns on the surface created by the subsequent bounces. Their results show that already after a few bounces the wavelengths close to the Faraday wavelength are dominant, while the others quickly dissipate. Moreover, they show that the wave pattern acts as a 'memory' containing the information from previous bounces (Labousse et al 2014).

\subsection{Relation with quantum mechanics}

The macroscopic droplet and the wave that supports it show some interesting behavior, with resemblance to the wave-particle duality in quantum mechanics. When barriers are placed in or nearby the path of the walker, the walker behaves as a combination of a wave and a particle. Although the system is macroscopic and hence not quantum-physical, the similarities can be exploited to explain that systems can show this kind of duality.

As an example, the walker system can be used to demonstrate wave diffraction in a single slit experiment (see figure 1): when a bouncing droplet is sent through a slit, it will change its direction upon passing through the slit. When the direction at which multiple walkers leave the slit is measured and added, a diffraction pattern is measured that would be similar to the one obtained when plane waves pass through the slit.

Whereas this observed behavior is clearly an interesting example of wave-particle duality, care should be taken in showing the limits of this particular analogy with quantum mechanics. First, as mentioned above, this is a macroscopic system where the velocity and position of the walkers is known at all times, in obvious contrast with quantum mechanics where accurate simultaneous measurement of position and momentum is excluded. Second, the role of the wave that supports the droplet is obviously different from a quantum mechanical wave. In quantum mechanics, the wave represents a probability amplitude, meaning that its squared amplitude denotes the probability density of the particle being at a certain position. In the case of the walker the waves form a kind of supporting structure for the bouncing droplet. This means that the wave shows behavior similar to the pilot wave interpretation of the de Broglie-Bohm theory (Bohm 1952a, 1952b). This interpretation considers the motion of quantum particles to be guided by a single associated pilot wave, described by the Schrödinger equation. This is in contrast with the Copenhagen interpretation, in which a measurement collapses the wave function to force the system to take one state explicitly. In the Bohm interpretation, the guiding wave is already present prior to any measurement. The randomness of the outcome of experiments in pilot-wave theory is therefore caused by the initial conditions and not by the dynamics.

The latter is true for the walker system: small variations in the initial conditions of the system cause the probabilistic behavior of the walkers. Because quantum physics education teaches the Copenhagen interpretation rather than the de Broglie-Bohm interpretation, care should be taken not to overstretch the walker analogy. A strong emphasis on the relation of the walker system with quantum mechanics, could lead to an implicit acceptance of the pilot wave interpretation. Although these two important differences exist between walkers and quantum mechanical experiments, walkers are suitable to display the basic principles of wave particle duality, and hence can function as an analogy for this phenomenon. 


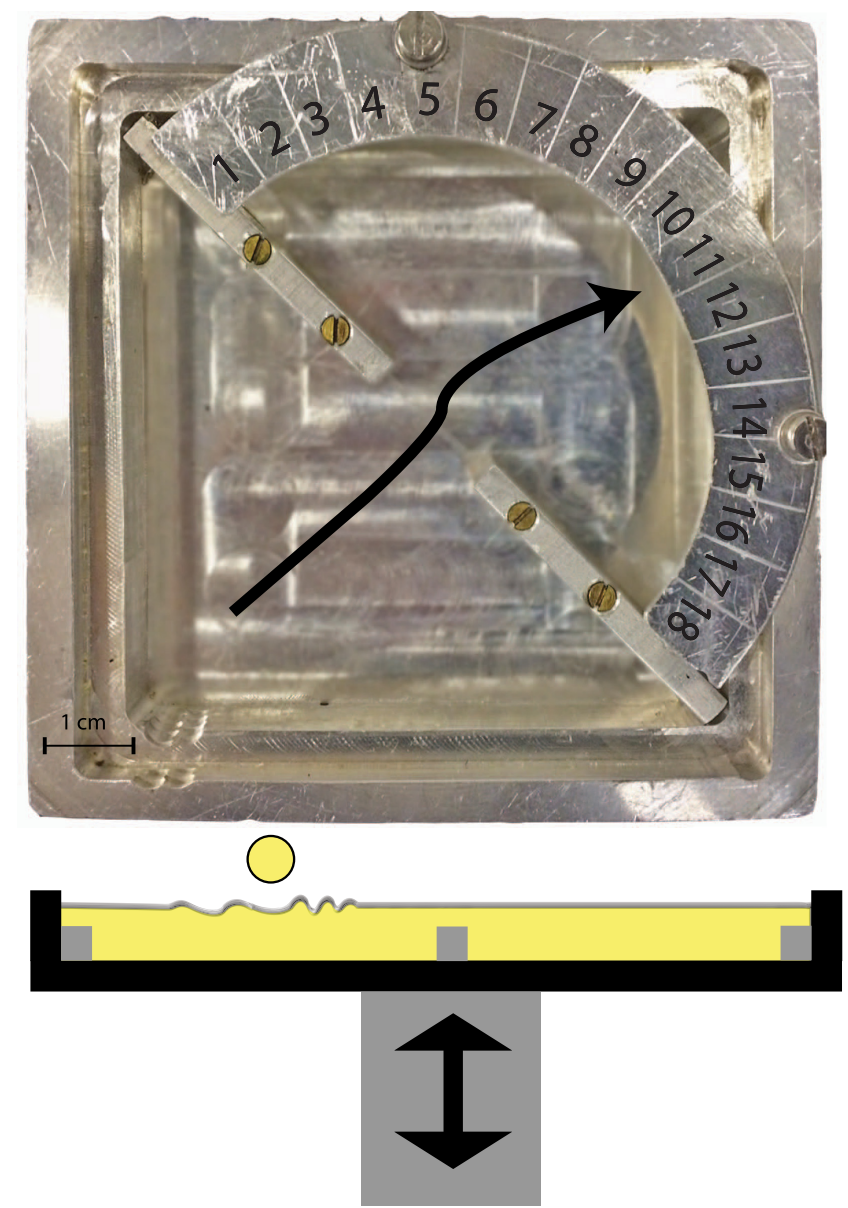

Figure 3. Top and side view of the oil bath for bouncing droplets. Droplets are generated in the lower left-hand side. The arrow indicates a possible droplet trajectory. After the droplet passes through the opening, students record the section in which the droplet arrives. Each section covers 10 degrees. The side view shows that the liquid bath has a uniform depth, with a barrier and ridge along the edges covered by a thin layer of oil.

\subsection{Experimental design}

An experimental setup was constructed following the description by Couder et al (Couder and Fort 2006). A sketch and photograph of the setup are shown in figure 3. An aluminum basin is mounted on top of a linear actuator. The actuator shakes the basin vertically with adjustable acceleration and frequency. The design used in the current work is suited for the single-slit diffraction experiment. Different designs are possible to perform other experiments (Couder et al 2010). Previous research used silicon oil, which can be ordered at a specified viscosity. One drawback is that silicon oil is expensive and notoriously difficult to remove from any surface. Soybean oil was therefore used as a cheap and widely available alternative, which can be obtained from the local supermarket and can be removed using water and a mild detergent. All fluid properties were mentioned in section 2.1. The design of the basin is 


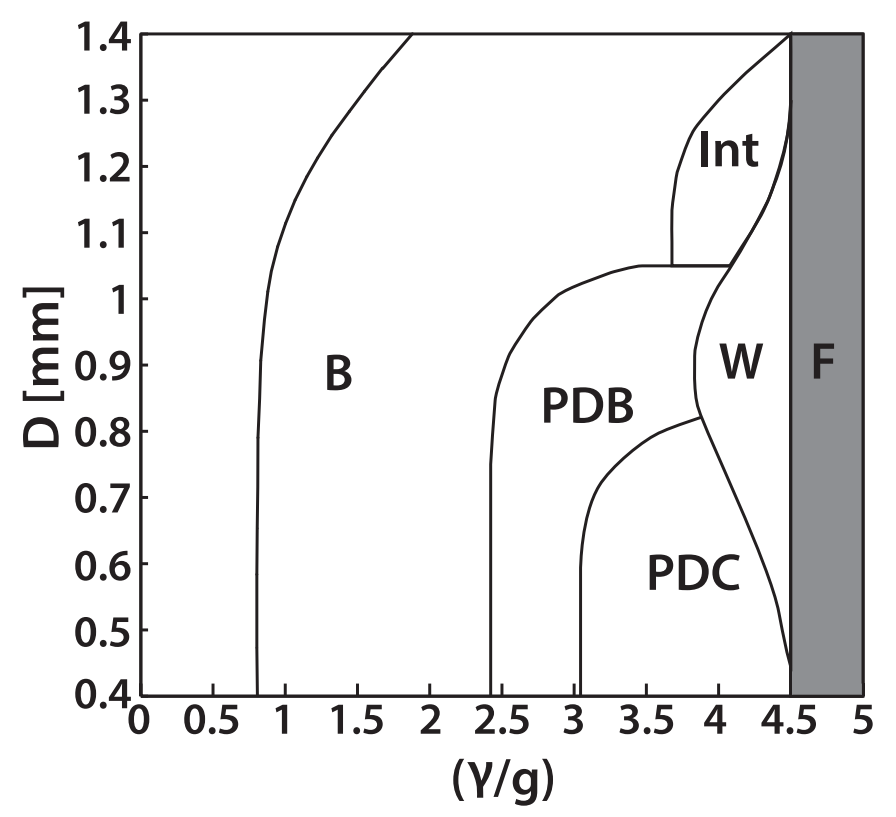

Figure 4. Parameter space for acceleration and droplet size $\mathrm{D}$, indicating various behavior, including bouncing (B), period doubling to bouncing (PDB) and period doubling to chaos (PDC), intermittent behaviour (Int) and the desired walker regime $(\mathrm{W})$. The gray right border indicated with $\mathrm{F}$ indicates the onset of Faraday waves at higher acceleration. Results are shown here for silicon oil $(\mu=50 \mathrm{mPa} . \mathrm{s})$ and a bath oscillation frequency of $50 \mathrm{~Hz}$. Reproduced with permission from Protière et al (2006).

crucial, because the depth of the oil bath, the type of oil, the size of the droplets, the shaking frequency and the slit-width are all interrelated. In the present work, the basin, milled from a single piece of aluminum, had internal dimensions of $80 \times 80 \times 15 \mathrm{~mm}^{3}$. A small ridge with a width of $5 \mathrm{~mm}$ was made around the edges, with the same height as the barriers $(10 \mathrm{~mm})$. The two aluminum barriers were detachable and placed diagonally, as shown in figure 3 . These barriers were $4 \mathrm{~mm}$ wide and $10 \mathrm{~mm}$ high. As it is advised to use a slit with a width of about three times the Faraday wavelength (Couder and Fort 2006), the distance $d$ between the barriers was $d=18 \mathrm{~mm}$. The basin was filled with $70 \mathrm{ml}$ of oil to obtain a liquid depth of $11 \mathrm{~mm}$. The barriers and ridge were thus covered by $1 \mathrm{~mm}$ of oil, required to reflect the walkers away from the walls to avoid collision of the droplets and the wall. The rigidity of the basin was found to be important: flexing and bending of the basin during oscillation affects the local amplitude of oscillation, marring the experiment. The basin also needs to be properly leveled, as any tilt in the basin floor will create a non-uniform liquid depth. The basin was connected to an actuator (TiraVib 50301) that could be set to the desired oscillation frequency and acceleration. The values used were $50 \mathrm{~Hz} \pm 2 \mathrm{~Hz}$, with an acceleration of $4-5 \mathrm{~g}$. The range of frequencies in this experiment, $20-100 \mathrm{~Hz}$, allows for replacing the professional shaker and electronics by a shaker constructed from audio speakers and amplifiers. This has recently been done successfully by a group of high school students, with assistance from the authors. Small droplets were generated by swiftly dipping a thin tip, e.g. a needle or a toothpick, in and out of the liquid. Droplets of $\approx 1 \mathrm{~mm}$ were found to be stable and display the desired walker behavior for the parameters used. Supplementary movie 2 shows a high speed recording of this process. 


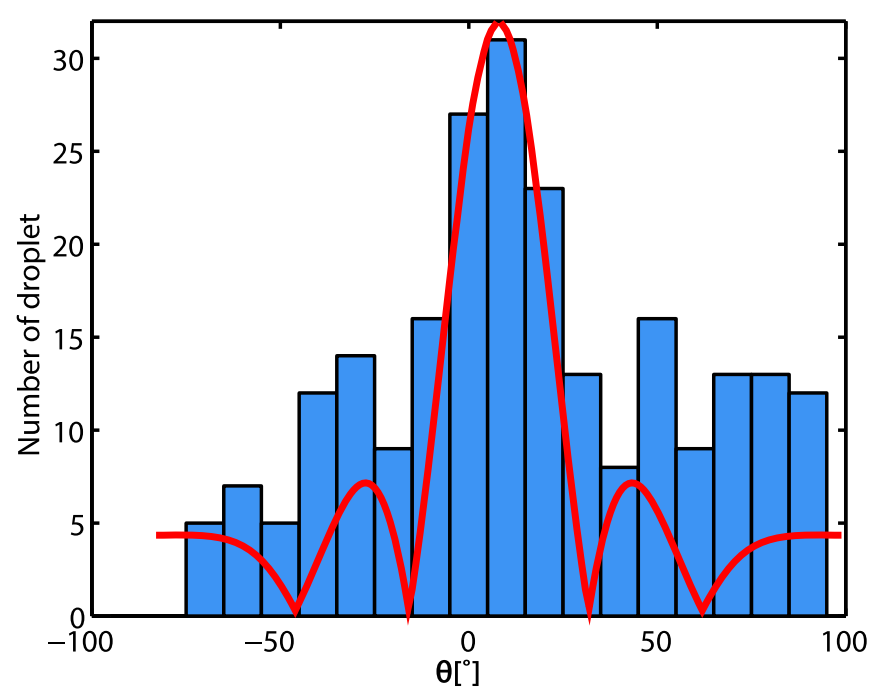

Figure 5. Histogram showing 233 data points collected by the students in the experiment. The expected distribution (equation (3)) is plotted in red, using an effective slit width $d=22 \mathrm{~mm}$.

\subsection{Setting the right parameters}

Bouncing droplets are easily formed for a wide range of droplet sizes and liquids, bath frequencies and accelerations. The desired walkers are only formed in a much narrower parameter space. The acceleration was set just below the threshold for onset of Faraday instability as shown in figure 4. This allows for more droplet sizes behaving as walkers. The value of the acceleration had to be adjusted regularly during the experiment to maintain good results.

\section{Pilot application in the classroom}

After the setup was constructed and tested, a group of ten high-school students (ages ranging from 16 to 18 years old) was invited to perform the single slit experiment. An introductory lesson was given to introduce or remind the students of the main concepts involved. In this lesson wave-particle duality was introduced by showing diffraction grid patterns and discussing single and double slit experiments. Single-particle diffraction patterns were shown and explained. Excellent applets are available to support this introduction (University of Colorado 2014). One week later, the experimental setup was prepared for the students. The experiment was executed in a laboratory space at the university. After a brief introduction, the students were divided into five pairs; each pair worked for 10-15 minutes on the experiment. One student in each pair focused on generating bouncing droplets by dipping a needle into the surface. The other student checked the trajectories of the walkers and wrote down the section in which the walkers arrived after passing through the single slit, see figure 3. After 5 minutes, they switched tasks. The students were observed by one of the authors, who supervised the experiment. The supervisor also took notes on the students' behavior, and recorded remarks made by the students. Directly after the experiment, each pair of students was interviewed. The interviews were videotaped, and transcribed later on to score the students' understanding 
(University of Victoria 2014). After the experiments, the students were assembled in a classroom. The observations of droplet trajectories by the individual groups were combined to form a probability histogram. This histogram, shown in figure 5 shows the number of droplets as a function of the angle of diffraction $\theta$, and is compared to the expected distribution

$$
N=\left|\operatorname{sinc}\left(\frac{d \pi \sin (\theta)}{\lambda}\right)\right|
$$

which is plotted in the same figure, taking a slit width $d=22 \mathrm{~mm}$. This is somewhat wider than the $18 \mathrm{~mm}$ at which the slit was constructed, possibly because the barriers had rounded edges, leading to a larger effective width of the slit. Some skewness in the histogram can be observed, likely due to improper alignment of the liquid bath. It is noteworthy that this skewness and misalignment was noted by some of the students, and coupled to 'one part of the liquid being wavy'.

\subsection{Observations and discussion}

Giving each student a task worked well, although most students focused strongly on performance of the prescribed task, enthusiastically urging the droplets to pass through the slit. This was confirmed by answers given in the interview conducted directly after the experiment. Students referred mostly to performing the tasks: 'Generating droplets that had to go through the slit'. However, two groups of students remarked that they were 'measuring the behavior of the droplets' and 'checking for diffraction patterns of arriving droplets'. The focus on the task of 'making droplets', caused many of the students to overlook other interesting behavior. Interaction between droplets, droplets hopping over the barrier and reflection from the walls was often observed by the supervising author, but not by the students. The focus on a recipe is a well-known effect that can occur in practicals (Millar 2010). Most students may also lack an adequate framework of reference, which results in the unexpected going unnoticed. After the experiment, and upon asking, all students showed that they understood that diffraction patterns were looked for. All of them were also able to recognize that the data they collected actually resembled a diffraction pattern. More and better explanation of the analogy with other slit experiments and what is observed in the experiment is likely to be advisable as $50 \%$ of the students thought that either the wave or the droplet was responsible for the diffraction pattern. The other $50 \%$ of the students saw correctly that it was in fact the combination of both droplet and the wave. Future work should involve a larger number of students which will allow for pre- and post-test application of concept tests to show growth in understanding (McKagan et al 2010).

\section{Conclusion}

The exciting and relatively new 'bouncing droplet' experiment provides a way to give students a glimpse of the combination of simultaneous particle and wave behavior. The experiment is stable enough to be performed by students in a classroom setting. Students worked in pairs and collected data from the single slit experiment. Their combined data clearly showed a diffraction pattern. Discussions with students showed that it is important to guide learners as the simultaneous particle and wave behavior is not automatically recognized by all. The next step is to bring the experimental setup to more classrooms in a robust version. In doing this, we will provide teachers with hints for introducing the experiment, and for 
follow-up peer and classroom discussions. This will help ensure that student understanding of duality is scaffolded as intended.

\section{Acknowledgments}

Detlef Lohse and Michel Versluis are acknowledged for support and encouragement of this publication. Harold Zandvliet is acknowledged for support, helpful discussions, and revision of the manuscript. This work is supported by NanoNextNL and Netherlands Organisation for Scientific Research (NWO).

\section{References}

Bohm D 1952a A suggested interpretation of the quantum theory in terms of 'hidden' variables: I. Phys. Rev. 85 166-79

Bohm D 1952b A suggested interpretation of the quantum theory in terms of 'hidden' variables: II. Phys. Rev. 85 180-93

Bush J W 2015 Pilot-wave hydrodynamics Ann. Rev. Fluid Mechan. 47 269-92

Couder Y, Boudaoud A, Protière S and Fort E 2010 Walking droplets: a form of wave-particles duality at macroscopic scale Euro Phys. News 41 14-8

Couder Y and Fort E 2006 Single-particle diffraction and interference at a macroscopic scale Phys. Rev. Lett. 97154101

Couder Y and Fort E 2012 Probabilities and trajectories in a classical wave-particle duality J. Phys.: Conf. Ser. 361012001

Eddi A, Sultan E, Moukthar J, Fort E, Rossi M and Couder Y 2011 Information stored in Faraday waves: the origin of a path memory J. Fluid Mech. 674 1-31

Hammond E G, Lawrence A J, Su C, Wang T, White A and Pamela J 2005 Bailey's Industrial Oil and Fat Products (Hoboken, NJ: John Wiley \& Sons, Inc)

Labousse M, Perrard S, Couder Y and Fort E 2014 Build-up of macroscopic eigenstates in a memorybased constrained system New J. Phys. 16113027

McKagan S B, Perkins K K and Wieman C E 2010 Design and validation of the quantum mechanics conceptual survey Phys. Rev. ST Phys. Educ. Res. 6020121

Millar R 2010 Analysing Practical Science Activities to Assess and Improve their Effectiveness (York: University of York Science Education Group) www.stem.org.uk/elibrary/resource/33677

Perrard S, Labousse M, Miskin M, Fort E and Couder Y 2014 Self-organization into quantized eigenstates of a classical wave-driven particle Nat. Commun. 53219

Pol H J, Krijtenburg K, Rossum A N and van Joolingen W R 2015 On the double-slit-experiment; applet versus demonstration MPTL 2015: 20th Int. Conf. on Multimedia in Physics Teaching and Learning (LMU Munich, 9-11 September 2015) poster session contribution P04

Protière S, Boudaoud A and Couder Y 2006 Particle-wave association on a fluid interface J. Fluid Mech. 554 85-108

Protière S, Couder Y, Fort E and Boudaoud A 2005 The self organisation of capillary wave sources $J$. Phys.: Condens. Matter. 17 S3529-35

University of Colorado $2014 \mathrm{https}$ //phet.colorado.edu/en/simulations/category/physics

University of Victoria 2014 www.coun.uvic.ca/learning/exams/blooms-taxonomy.html 